\title{
Naturheilkundliche Therapie beim kranken Kind
}

\author{
Viele Eltern wünschen, dass ihr Kind naturheilkundlich behandelt wird, da \\ sie annehmen, dass es sich hierbei um besonders verträgliche Therapiever- \\ fahren handelt. Grundsätzlich ist dies richtig, allerdings wird zunehmend \\ von verschiedenen Seiten gefordert, dass Wirksamkeit und Verträglichkeit \\ indikationsspezifisch belegt sind.
}

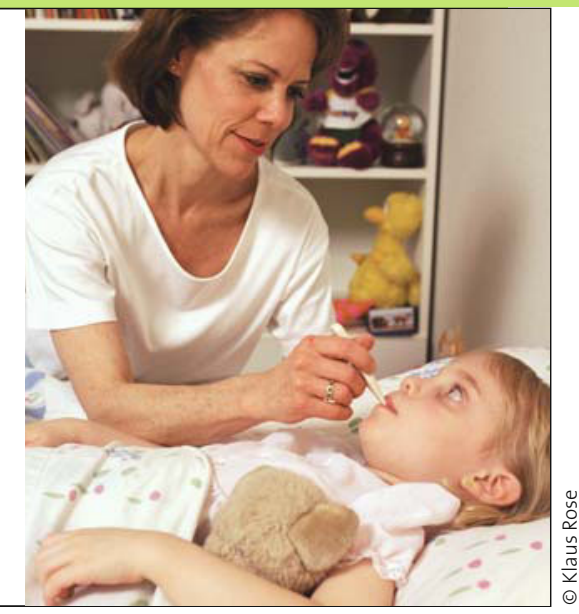

- Kinder unterscheiden sich bekanntlich hinsichtlich der Organfunktionen, aber auch psychologisch deutlich von den Erwachsenen, dementsprechend sind einige Besonderheiten zu beachten. Dennoch können fast alle Naturheilverfahren auch bei Kindern gut angewendet werden. Für Kinder spezifische Indikationen, die mit Naturheilverfahren behandelt werden können, sind Milchschorf, Nägelkauen, Enuresis, Schlafstörungen und Albträume, Unruhe und Aggressivität, Essunlust, Wachstumsstörungen und Lernschwierigkeiten. Bewährte Therapiekonzepte sollen in späteren Folgen dargestellt werden.

\section{Entspannungsverfahren}

Das Erlernen des autogenen Trainings ist etwa ab dem sechsten Lebensjahr möglich. Es kann durch seine spannungsreduzierende Wirkung bei vielen kindlichen Verhaltensstörungen eingesetzt werden, z.B. als adjuvante Therapie bei Nägelkauen, Enuresis, Schlafstörungen und Lernschwierigkeiten.

\section{Manuelle Therapie}

Empirisch sprechen durch Dysfunktionen im Bereich der Kopfgelenke bedingte Wachstumshemmungen und Sprachentwicklungsverzögerungen gut an. Bei Hüftdysplasien kann der Verlauf durch Korrektur einer Dysfunk- tion der Ileosakralgelenke günstig beeinflusst werden.

\section{Ordnungstherapie}

Ordnungstherapie ist bei chronischen oder rezidivierenden Erkrankungen als unterstützende Maßnahme von großem Wert. Kinder fühlen sich in Ritualen geborgen und sicher. Der Tag sollte deshalb rhythmisch gegliedert werden mit möglichst regelmäßigen Zeiten des Aufstehens, der Mahlzeiten und des Zubettgehens. Auf eine gesunde Ernährung und eine regelmäßige Bewegung bei viel Aufenthalt im Freien ist großer Wert zu legen.

\section{Nahrungsergänzungsmittel}

Hier sollte man bei Kindern sehr zurückhaltend sein und sie nur verabreichen, wenn ihre Indikation sorgfältig geprüft wurde. Kinder gewöhnen sich sonst schnell daran, Zubereitungen, die wie Arzneimittel aussehen, zur Lösung von Problemen wie z. B. Befindlichkeitsstörungen einzunehmen, wodurch einer späteren unkritischen Einnahme von Medikamenten oder gar Drogen Vorschub geleistet werden kann.

\section{Phytotherapie}

Die Therapie mit mild wirkenden Arzneidrogen wird traditionell in der Kinderheilkunde bei Befindlichkeitsstö- rungen und leichten Erkrankungen häufig eingesetzt. Es gibt gerade für Kinder sehr gut geeignete Anwendungsformen wie Teemischungen, Salben, Frischpflanzenpresssäfte, Suppositorien und Bäder. Zu beachten ist allerdings, dass die auf den Beipackzetteln angegebenen Dosierungen zumeist für Erwachsene gelten, dementsprechend müssen sie für die unterschiedlichen Altersstufen reduziert werden. Es gibt aber auch pflanzliche Fertigarzneimittel mit bereits angegebener Dosierung für Kinder.

Bei Infektionen des Respirationstraktes, des Gastrointestinaltraktes und der Harnwege ist Phytotherapie sehr sinnvoll, da für viele Arzneipflanzen (z. B. Eibischwurzel, Kamillenblüten, Eisenkraut) nachgewiesen wurde, dass sie die unspezifische Immunabwehr unterstützen, die bis zum elften Lebensjahr die dominierende Form ist. Des Weiteren ist Phytotherapie zur Behandlung von Schlafstörungen und leichteren psychischen Störungen des Kindesalters gut geeignet. Die geringe Nebenwirkungsrate und die hohe Akzeptanz bei den Eltern bewirken zudem eine gute Compliance.

- Prof. Dr. med. Karin Kraft Lehrstuhl Naturheilkunde, Universität Rostock, E-Mail: karin.kraft@med.unirostock.de 